\title{
ANALYSIS OF THE ELASTOSTATIC SPECIFIC STIFFNESS OF 2D STRETCHING-DOMINATED LATTICE MATERIALS
}

\author{
Mostafa S.A. Elsayed and Damiano Pasini \\ Department of Mechanical Engineering, McGill University, Montreal, QC, H3A 2K6, \\ CANADA
}

\section{ABSTRACT}

This paper presents a matrix-based procedure to characterize the specific stiffness properties of 2D lattice materials with any arbitrary cell topology. Unlike previous works, the current study automates the analysis process to include lattice materials whose unit cell has elements extending between adjacent cells and thus intersecting their envelopes. The main challenge in the analysis of this periodic lattice structures is that the unit cell does not have the full information concerning its nodal kinematic and static periodicity. For this reason, we introduce the Dummy Node Scheme, which enables the analysis of lattice material with any cell topology.

The lattice material is modelled here as a pin-jointed infinite micro-truss structure. The results of the determinacy analysis are used to distinguish between the bendingdominated and the stretching-dominated behaviours of the material. The Cauchy Born Hypothesis is used to homogenize the lattice material properties by formulating the microscopic lattice nodal deformations in terms of the material macroscopic strain field. This formulation, in turn, is used to express the microscopic element deformations in terms of the macroscopic strain field, from which the material macroscopic stiffness properties are derived. In this process, the Dummy Node Scheme is a necessary step to construct the nodal periodicity within the unit cell, which is used to apply the Cauchy Born kinematic boundary condition to the nodal deformation wave functions. The procedure introduced in this paper is applied to 10 lattice topologies, five of which have unit cells with a square Bravais lattice symmetry and the other five have unit cells with a hexagonal Bravais lattice symmetry. Finally, charts representing the relative elastic moduli of the lattice material versus its relative density are developed. These charts assist the selection of the best topology of a stretching-dominated lattice material for a given application that requires a material with specific stiffness properties.

KEYWORDS: Lattice Material, Micro-truss, Bloch's Theorem, Dummy Node Scheme, Stretching-Dominated Lattice Material.

\section{INTRODUCTION}

A lattice material is a type of cellular material with periodic microstructure. The unit cell is the building block used to tessellate the space into a periodic modular pattern. An important condition to generate a consistent tessellation is that the unit cell should have a minimum level of symmetry, as defined by the Bravais lattice symmetry [1]. There are five Bravais lattice symmetries in 2D. In the current study, we consider only lattice unit cells with hexagonal and square Bravais lattice symmetries. Several 2D lattice materials with hexagonal as well square Bravais lattice symmetry have been introduced in literature [2- 5]. The former includes the regular fully triangulated lattice, the regular hexagonal lattice and the semi-regular Kagome' lattice, which have the modified Schläfli symbols of 
$3^{6}, 6^{3}$ and 3.6.3.6, respectively. The latter consists of the regular square lattice, the rectangular lattice and the double braced square lattice.

A lattice material can be classified into bending and stretching-dominated materials with respect to the microscopic failure mode of the unit cell elements. Microscopic structural analysis shows that the bending-dominated lattice material has a low nodal connectivity at the cell vertices, which results in a microscopic bending-dominated failure mode, where the cell elements collapse by bending stresses. This feature generates non-optimal mechanical properties where the element solid materials are not fully employed in the microscopic loading resistance. On the contrary, the stretching-dominated lattice material has a high nodal connectivity at the cell vertices, which results in microscopic stretchingdominated failure mode where the cell elements collapse by axial stresses, giving a much higher stiffness and strength per unit mass. For instance, the structural analysis of stretching-dominated lattice material shows that its stiffness and strength scale up with the density ratio of the lattice material to the solid material, $\bar{\rho}$; on the other hand, the stiffness and the strength of the bending-dominated material are governed, respectively by $\bar{\rho}^{2}$ and $\bar{\rho}^{3 / 2}$ [6]. The different scaling laws have a strong impact on the strength and stiffness of the material. For example, at $\bar{\rho}=0.01$, the stretching-dominated lattice material has superior static performance because it is a hundred times stiffer and ten times stronger than the bending-dominated material.

To distinguish between bending and stretching-dominated lattice materials, we resort to the analysis of the kinematic determinacy of the pin-jointed version of the lattice micro structure. Maxwell (1864) [7] set a rule for the minimum number of bars necessary for a pin jointed framework to be kinematically determinate; these minimum numbers of bars are, $(2 j-3)$ and (3j-6) in 2D and 3D frameworks, respectively, where $j$ is the number of joints within a finite framework. A framework with less number of bars than the minimum condition of Maxwell is a mechanism, unless its joints are set to be rigid; in this case the framework behaviour is bending-dominated. Calladine [8] and Pellegrino [9, 10] reviewed the linear-algebraic basis of Maxwell's rule using the fundamental subspaces of the equilibrium and the kinematic matrices of a pin jointed framework. As a result, they reformulated the problem to obtain the generalization of Maxwell's rule, which includes information about the states of self-stress and the states of internal mechanisms within the framework. A state of self-stress is the vector of element forces generated within an unloaded framework; on the other hand, a state of internal mechanism is the vector of joint displacements corresponding to non-deforming elements. The generalized Maxwell's rule can be used to obtain an accurate prediction of the determinacy state of finite lattice structures in the form of a unit cell or a finite cluster of cells. Since the lattice material is structured at the microscale while its effective properties are homogenized at the macroscale, the analysis of the lattice material assumes a periodicity of the unit cell in an unbounded space. Therefore a complete determinacy analysis of lattice materials requires extending the analysis to the infinite lattice structure. Such an extension was proposed by Deshpande et al. (2001) [11] who examined the pinjointed mechanics of a restricted set of infinite-periodic lattice topologies. They considered only topologies wherein the joints are similarly-situated, i.e. the framework appears the same and in the same orientation regardless of the viewpoint. In 2D, these are the regular square and triangular lattices; in 3D, this set includes the regular octet-truss. The generalized Maxwell's rule, was used to prove that the necessary but not sufficient 
nodal connectivity, $Z$, of a structure to be stretching-dominated is $Z=4$ and $Z=6$ in $2 \mathrm{D}$ and $3 \mathrm{D}$, respectively. On the other hand, the sufficient nodal connectivity was proven to be $Z=6$ and $Z=12$ in 2D and 3D, respectively. More recently, Hutchinson (2004) [4] used the Bloch's theorem for modeling periodic waves in an infinite lattice structure with any Bravais symmetry. His analysis focused mainly on the case where the cell elements of the lattice share their end points with those of the cell envelope. However, a procedure to analyze lattice materials whose unit cells cell elements intersect their envelopes need to be formulated.

In this work, the analysis of lattice materials is extended to consider the case where some of the cell elements do not intersect the cell envelope at their end joints. For this purpose, we introduce the Dummy Node Scheme, which consists of adding dummy nodes at the points of intersection between the microscopic cell elements and the cell envelope. These dummy nodes are used to generate the kinematic and the equilibrium matrices of the unit cell finite microstructure. In addition, they are also used to generate an explicit expression for the microscopic nodal deformations in terms of a macroscopic hypothetical homogeneous strain field, as assumed by the Cauchy-Born hypothesis [12]. The degrees of freedom associated with the hypothetical dummy nodes are later removed from the generated matrix systems. This procedure is integrated in a matrix formulation for a comprehensive structural analysis of different lattice topologies. The results are then plotted in design charts that help to gain insight into the stiffness generated by the considered cell topology.

Organized in five sections, the paper introduces the theoretical analysis and the description of the systematic procedure in section two. In the third section, this procedure is applied to the different topologies with hexagonal and square Bravais lattice symmetries. The forth sections section compares the characterized stiffness properties before presenting the concluding remarks in the last section.

\section{ANALYSIS}

Notions of solid-state physics can help in solid mechanics to examine the characteristics of lattice materials. Principles of symmetry, for example, are often used in solid-state physics to simplify the formulation of the governing law of crystals. In this paper, we adopt the classical notion of a crystal structure, which can be described by introducing two characterizing parameters as [1]:

$$
\text { Crystal }=\text { Lattice }+ \text { Bases }
$$

The lattice is defined as a translational infinitely periodic arrangement of points [13, 14]. When periods of the unit cell are perfectly stacked in two or three dimension, the space is told to be tessellated. The bases are the mathematical representation for the physical constituents that are repeated in every cell translation.

In continuum mechanics, a lattice material can be characterized by adopting the above definition. The cell envelop, which defines the structure periodicity, is described in mathematical terms by the lattice translational symmetry primitive bases, $\vec{a}_{k}$, where $k \in\{1, . ., n\}$ and $n=2$ or $n=3$ in $2 \mathrm{D}$ or $3 \mathrm{D}$, respectively. The set of bases, representing the physical structure, contains two groups, namely, the joint bases group and the bar bases group. 


\section{Fig (1) 2D Square Lattice Structure}

Figure (1) illustrates this concept, as applied to the square lattice. Fig (1-a) shows the microscopic crystal structure of the lattice material, where two candidate unit cells (A) and (B) are shown (within the dotted envelope). Fig (1-b) shows the lattice translational symmetry primitive bases $\vec{a}_{1}$ and $\vec{a}_{2}$. Figures (1-c) and (1-d) illustrate the physical structure bar position vectors, $\vec{b}_{m}$, and joint position vectors, $\vec{j}_{l}$, of the candidate unit cells, (A) and (B), respectively, where $m \in\{1,2, \ldots b\}$ and $l \in\{1,2, \ldots j\} . b$ and $j$ are the total number of bars and joints within the unit cell structure, respectively.

\section{II.1 Unit Cell Determinacy Analysis}

Following the approach by Calladine and Pellegrino [8-10], we consider a finite truss structure that consists of $j$ total joints connected by $b$ bars. The $b$ bar tension forces and displacement deformations are assembled into vectors $t$ and $e$, respectively. On the other hand, the $n j$ components of external force and joint displacements are assembled into vectors $f$ and $d$, respectively. The equilibrium and the kinematic systems of the truss structure are expressed as:

$$
\begin{aligned}
& \text { A.t } t=f \\
& \text { B. } d=e
\end{aligned}
$$

where $\boldsymbol{A} \in R^{(n j) \times b}$ is the equilibrium matrix and $\boldsymbol{B} \in R^{b \times(n j)}$ is the kinematic matrix. The formulation of the equilibrium and the kinematic matrices for the unit cell shown in Fig (1-c) is straightforward. However, to generate the equilibrium and the kinematic matrices of the unit cell illustrated in Fig (1-d), we need to introduce an alternative procedure, as explained in the following.

\section{A. Dummy Node Scheme}

The dummy node Scheme is introduced to deal with lattice structures containing structural elements extending between adjacent unit cells. The derivation and theoretical analysis of the dummy nodes is detailed in appendix A. Here, we describe the steps required to apply the dummy node Scheme.

Step 1: Hypothetical dummy nodes are introduced at the intersection points between the microscopic cell elements, extending between neighbouring unit cells, and the cell envelope. The kinematic and the equilibrium matrices of the finite microstructure are then reformulated to take into account the dummy nodes.

Step 2: Once the kinematic and the equilibrium systems are formulated, the degrees of freedom associated with the dummy nodes are eliminated from the generated matrices. The degrees of freedom associated with the dummy nodes are expressed in the row space of the equilibrium matrix, given in eqn (2a), as well as in the column space of the kinematic matrix, given in eqn (2b). To eliminate the degrees of freedom associated with the dummy nodes, all modes in the row space of the equilibrium matrix and the column space of the kinematic matrix that are associated with the dummy nodes are eliminated. 
The same elimination technique is applied to the nodal force and the nodal displacement vectors.

As an example, consider the unit cell, (B), shown in Fig (1-d). The above steps are applied as follows:

Step 1: Four dummy nodes are defined for the unit cell (B) as shown in Fig (2). It should be noted that in this case the dummy node position vectors are coincident with the position vectors of the bars that intersect with the cell envelope and the total group of joint position vectors now includes also the dummy nodes position vectors. Using this new group of joint position vectors along with the group of bar position vectors, the equilibrium and the kinematic matrices of the unit cell structure can be formulated as:

$$
\begin{aligned}
& {\left[\begin{array}{cccc}
-1 & 0 & 1 & 0 \\
0 & -1 & 0 & 1 \\
0 & 0 & -1 & 0 \\
0 & 0 & 0 & 0 \\
0 & 0 & 0 & 0 \\
0 & 0 & 0 & -1 \\
1 & 0 & 0 & 0 \\
0 & 0 & 0 & 0 \\
0 & 0 & 0 & 0 \\
0 & 1 & 0 & 0
\end{array}\right]\left[\begin{array}{l}
t_{1} \\
t_{2} \\
t_{3} \\
t_{4}
\end{array}\right]=\left[\begin{array}{l}
f_{1 x} \\
f_{1 y} \\
f_{d 1 x} \\
f_{d 1 y} \\
f_{d 2 x} \\
f_{d 2 y} \\
f_{d 3 x} \\
f_{d 3 y} \\
f_{d 4 x} \\
f_{d 4 y}
\end{array} \mid\right.} \\
& \left.\left[\begin{array}{cccc}
-1 & 0 & 1 & 0 \\
0 & -1 & 0 & 1 \\
0 & 0 & -1 & 0 \\
0 & 0 & 0 & 0 \\
0 & 0 & 0 & 0 \\
0 & 0 & 0 & -1 \\
1 & 0 & 0 & 0 \\
0 & 0 & 0 & 0 \\
0 & 0 & 0 & 0 \\
0 & 1 & 0 & 0
\end{array}\right] \mid \begin{array}{l}
d_{1 x} \\
d_{1 y} \\
d_{d 1 x} \\
d_{d 1 y} \\
d_{d 2 x} \\
d_{d 2 y} \\
d_{d 3 x} \\
d_{d 3 y} \\
d_{d 4 x} \\
d_{d 4 y}
\end{array}\right]=\left[\begin{array}{l}
e_{1} \\
e_{2} \\
e_{3} \\
e_{4}
\end{array}\right]
\end{aligned}
$$

where $f_{d}$ and $d_{d}$ are dummy node forces and deformations, respectively.

Step 2: the degrees of freedom associated with the dummy nodes are now eliminated from the matrix systems of eqn (3), which results in: 


$$
\begin{gathered}
{\left[\begin{array}{cccc}
-1 & 0 & 1 & 0 \\
0 & -1 & 0 & 1
\end{array}\right]\left[\begin{array}{l}
t_{1} \\
t_{2} \\
t_{3} \\
t_{4}
\end{array}\right]=\left[\begin{array}{l}
f_{1 x} \\
f_{1 y}
\end{array}\right]} \\
{\left[\begin{array}{cc}
-1 & 0 \\
0 & -1 \\
1 & 0 \\
0 & 1
\end{array}\right]\left[\begin{array}{l}
d_{1 x} \\
d_{1 y}
\end{array}\right]=\left[\begin{array}{l}
e_{1} \\
e_{2} \\
e_{3} \\
e_{4}
\end{array}\right]}
\end{gathered}
$$

Equation (4) shows, respectively, the equilibrium and the kinematic systems of the unit cell shown in Fig (1-d).

For the determinacy analysis of the structure, we resort to the classical four fundamental vector subspaces of the kinematic and the equilibrium matrices [9].

\section{Fig (2) Dummy nodes ( $\square$ ) added to the unit cell}

It should be noted that, for the determinacy analysis of the unit cell finite structure, the computation of the four fundamental subspaces must be applied to the equilibrium and the kinematic matrices that include the degrees of freedom associated with the dummy nodes, e.g. the matrices given in eqn (3) for the square lattice. The reason for this is that the elimination of the dummy nodes from the matrix system of the finite structure acts as the application of boundary conditions that fix the finite structure into a foundation which results in inaccurate results. However, for the determinacy analysis of the infinite lattice structure, the reduced forms of the kinematic and the equilibrium matrices, e.g. the matrices given in eqn (4) for the square lattice, are used.

\section{II.2 Infinite Structure Determinacy Analysis}

Hutchinson [4] first applied the Bloch's theorem to the determinacy analysis of the infinite lattice structure. The Bloch's theorem requires the definition of a set of parameters to describe a wave- function over the infinite lattice structure. The same approach is used in this work and the relevant lattice parameters are briefly defined here.

\section{A. Direct Translational Bases}

The lattice translational symmetry primitive bases, $\vec{a}_{k}$, are referred to as the direct translational bases, which govern the process of the cell tessellation.

\section{B. Direct Translational Vector}

A direct translational vector is formulated as a linear combination of direct translational bases; this vector is used to translate the reference unit cell to any other cell in the space of the lattice. The direct translational vector is formulated as:

$$
\vec{R}=\sum_{k=1}^{n} m_{k} \vec{a}_{k}
$$


Where $m_{k}$ is any set of integers and $n$ is the dimensional space of the lattice. It should be noted that the direct translational vector is the Bravais lattice vector spanned over a set of cells in the lattice space.

\section{Position Vectors}

By using the definition of bar and joint bases of the reference unit cell envelope, along with the definition of the direct translational vector, the position vectors of bars and joints of the whole crystal structure can be formulated as:

$$
\begin{aligned}
& p_{l}=j_{l}+\vec{R}=j_{l}+\sum_{k=1}^{n} m_{k} \vec{a}_{k} \forall l \in\{1, \ldots, J\}, \\
& k \in\{1, \ldots, n\}, n=2 \text { in } 2 D \text { and } n=3 \text { in } 3 D \text { and } m_{k} \in Z \\
& q_{m}=b_{m}+\vec{R}=b_{m}+\sum_{k=1}^{n} m_{k} \vec{a}_{k} \forall m \in\{1, \ldots, B\}, \\
& k \in\{1, \ldots, n\}, n=2 \text { in } 2 D \text { and } n=3 \text { in } 3 D \text { and } m_{k} \in Z
\end{aligned}
$$

where $p_{l}$ and $q_{m}$ are the joints and the bars position vectors, respectively. $J$ and $B$ are, respectively, the number of independent joints and the number of independent bars, within the reference unit cell envelope.

\section{Direct Lattice}

The direct lattice contains the set of independent bar and joint bases, over the reference unit cell envelope, spanned over the infinite periodic lattice structure by their position vectors. This set of infinite bases is called the direct lattice. To determine the independent set of bar and joint bases over the reference unit cell, we verify the dependency of the bases within the reference unit cell through the relation:

$$
V_{i-1}=V_{i}+\sum_{k=1}^{n} \hat{x}_{k} \vec{a}_{k}
$$

where $x_{k} \in\{-1,0,1\}$ is a unit translation vector, If $V_{i-1}$ and $V_{i}$ belong to the joint position vectors, then $V_{i} \equiv j_{l} \& i \equiv l \in\{1, \ldots, J\}$ and if they belong to the bar position vectors, then $V_{i} \equiv b_{m} \& i \equiv m \in\{1, \ldots, B\}$. The dependency information is used later to modify the wavefunction over the reference unit cell to generate the periodic wave- function over the infinite lattice.

\section{E. Reciprocal Lattice}

The reciprocal lattice is itself a Bravais lattice introduced to describe the lattice in terms of primitive vectors. The advantage of resorting to the reciprocal lattice is to discretize the continuous space of the lattice into a discrete summation of modes at which the lattice performance can be examined. The reciprocal lattice can be represented by the primitive vectors $\vec{b}_{1}$ and $\vec{b}_{2}$, which are defined as: 


$$
\vec{b}_{i} \bullet \vec{a}_{j}=2 \pi \delta_{i j}
$$

where $\vec{a}_{j}$ and $\vec{b}_{i}$ are the direct and the reciprocal lattice bases, respectively, and $i, j \in\{1,2\}$ in 2 D. $\delta_{i j}$ is the Kronecker delta symbol that satisfies:

$$
\delta_{i j}= \begin{cases}0 & \text { for } i \neq j \\ 1 & \text { for } i=j\end{cases}
$$

Thus, the translational vectors of the reciprocal lattice are defined as:

$$
\omega=\omega_{1} \vec{b}_{1}+\omega_{2} \vec{b}_{2} \quad \forall \omega_{1}, \omega_{2} \in[0,1) \subset Q
$$

where $\omega_{1}$ and $\omega_{2}$ are the covariant components of $\omega$ with respect to the basis $\vec{b}_{1}$ and $\vec{b}_{2}$ and Q is the set of all rational numbers. $\omega_{1}$ and $\omega_{2}$ are defined over the open subset of Q from zero to near unity in agreement with the Bloch's theorem [4], described in the following section.

\section{F. Bloch's theorem}

The Bloch's theorem is used to extend the determinacy analysis of the unit cell to the unbounded periodic lattice.

\section{F.1 Bloch-wave mechanisms and States of Self Stress}

The Bloch's theorem is applied to define the propagation of a wave function over the infinite lattice structure. For nodal deformation functions, the generalized nodal displacement vectors $d\left(p_{l}, \omega\right) \in C^{2}$ can be expressed over the entire lattice as a wave function of the form:

$$
d\left(p_{l}, \omega\right)=d\left(j_{l}+\vec{R}, \omega\right)=d\left(j_{l}, \omega\right) e^{2 \pi i \omega \vec{R}} \forall l \in\{1,2, \ldots, J\}
$$

where $J$ is the number of independent nodes within the unit cell envelope, $p_{l}=j_{l}+\vec{R}$ is the position vector of any node throughout the lattice and $\vec{R}$ is the Bravais cell vector of any unit cell through the entire lattice.

Similarly, for bar deformation functions, the generalized bar deformation vectors $e\left(q_{m}, \omega\right) \in C^{2}$ can be expressed over the entire lattice as a wave function of the form:

$$
e\left(q_{m}, \omega\right)=e\left(b_{m}+\vec{R}, \omega\right)=e\left(b_{m}, \omega\right) e^{2 \pi i \omega} \vec{R} \forall m \in\{1,2, \ldots, B\}
$$


where $B$ is the number of independent bars within the unit cell envelope and $q_{m}=b_{m}+\vec{R}$ is the position vector of any bar throughout the lattice.

To reduce the forms of the kinematic and the equilibrium matrices, we define transformation matrices for both bars and joints. This procedure makes use of the periodic boundary conditions defined over the unit cell $[16,17]$.

\section{F.2 Bars and Joints Transformation Matrices}

Consider the generic unit cell shown in Fig (3), by using the Bloch's theorem, the following relations can be obtained

Fig (3) generic unit cell with its periodic displacement boundary conditions

$$
\begin{gathered}
q_{R}=e^{\mu_{x}} q_{L}, q_{T}=e^{\mu_{y}} q_{B}, q_{R B}=e^{\mu_{x}} q_{L B}, \\
q_{L T}=e^{\mu_{y}} q_{L B}, q_{R T}=e^{\mu_{x}+\mu_{y}} q_{L B}
\end{gathered}
$$

Where $q$ is a generic nodal or element deformation functions, and $T, B, L$ and $R$ denote top, bottom, left and right, respectively. $\mu_{x}$ and $\mu_{y}$ are the wave numbers, derived from the reciprocal space of the lattice along with the dependency relations of the joints and elements bases, which are expressed as:

$$
\mu_{x}=2 \pi \omega_{1} i, \quad \mu_{y}=2 \pi \omega_{2} i
$$

Equation (12) can be arranged in matrix form as:

$$
\left[\begin{array}{c}
q_{I} \\
q_{B} \\
q_{T} \\
q_{L} \\
q_{R} \\
q_{L B} \\
q_{R B} \\
q_{L T} \\
q_{R T}
\end{array}\right]=\left[\begin{array}{cccc}
I & 0 & 0 & 0 \\
0 & I & 0 & 0 \\
0 & I e^{\mu_{y}} & 0 & 0 \\
0 & 0 & I & 0 \\
0 & 0 & I e^{\mu_{x}} & 0 \\
0 & 0 & 0 & I \\
0 & 0 & 0 & I e^{\mu_{x}} \\
0 & 0 & 0 & I e^{\mu_{y}} \\
0 & 0 & 0 & I e^{\mu_{x}+\mu_{y}}
\end{array}\right]\left[\begin{array}{c}
q_{I} \\
q_{B} \\
q_{L} \\
q_{L B}
\end{array}\right], \quad \text { or } q=T \tilde{q}
$$

where $T$ is the transformation matrix from the primitive cell degrees of freedom to the reduced cell degrees of freedom. The transformation matrices for the element deformations and the nodal displacements wave-functions are obtained such that:

$$
\begin{aligned}
& e=T_{e} \tilde{e} \\
& d=T_{d} \tilde{d}
\end{aligned}
$$


where $\tilde{e}$ and $\tilde{d}$ are the element deformations and the nodal displacements reduced vectors (periodic wave-function), respectively. $T_{e}$ and $T_{d}$ are the matrices that transforms respectively the full vectors of the periodic element deformations and nodal displacements to their respective reduced periodic vectors.

The technique described above to generate the transformation matrices is applied to the generic unit cell shown in Fig (3). The transformation matrices are generated taking into account the dependency relations of the bars and the joints bases. These dependency relations are computed by eqn (7). The key parameter in eqn (7) is the direct translational bases, $\vec{a}_{k}$, which is formulated through the lattice symmetry and the unit cell geometry. Details about the technique used to formulate the transformation matrices are given in appendix B.

Substituting eqns (15) into the kinematic matrix of the finite truss, $B d=e$, gives

$$
B T_{d} \tilde{d}=T_{e} \tilde{e}
$$

The transformation matrix $T_{e}$ is a complex non-square matrix, which can be inverted by multiplying $T_{e}$ by its conjugate transpose (the Hermitian transpose), $T_{e}^{H}$, such that:

$$
T_{e}^{H} B T_{d} \tilde{d}=T_{e}^{H} T_{e} \tilde{e}
$$

The multiplication of a complex matrix by its Hermitian transpose generates a block real matrix, $B_{e}$ as follows:

$$
T_{e}^{H} T_{e}=B_{e}
$$

Substituting eqn (18) into eqn (17) and inverting the real block matrix $B_{e}$ results in

$$
\left(B_{e}\right)^{-1} T_{e}^{H} B T_{d} \tilde{d}=\tilde{e}
$$

From eqn (19), the reduced kinematic matrix is expressed as:

$$
\tilde{B}=\left(B_{e}\right)^{-1} T_{e}^{H} B T_{d}
$$

The reduced kinematic and equilibrium matrices are fundamental to the determinacy state of the infinite lattice, which in turn can be analyzed by computing their four fundamental subspaces. This procedure enables to determine the independent sets of periodic mechanisms and periodic states of self-stress for the different wave vectors $\left(\omega_{1}, \omega_{2}\right)$ that are obtained from the irreducible first Brillouin Zone of the reciprocal lattice [18].

\section{II.3 Macroscopic Strain Generated by Microscopic Mechanisms}


The Bloch's theorem allows characterizing mechanisms corresponding to periodic joint displacement fields. To examine the macroscopic strain field generated by periodic mechanisms, we resort to the Cauchy-Born hypothesis [19-22].

\section{A. Cauchy- Born Hypothesis}

From the definition of the Cauchy-Born hypothesis [4], the infinitesimal displacement field of a periodic joint in a lattice structure can be formulated as:

$$
d\left(j_{l}+\vec{R},-\bar{\varepsilon}\right)=d\left(j_{l},-\bar{\varepsilon}=0\right)+\vec{\varepsilon} \cdot \vec{R}
$$

where $d\left(J_{l}, \bar{\varepsilon}=0\right)$ is the periodic displacement field of joint $j_{l}$. Assume that the periodic joints defined by the position vectors $j_{l}$ and $j_{l}+\vec{R}$, are the two periodic joints $i$ and $j$ within a lattice structure, then, eqn (21) can be formulated in matrix form as:

$$
\left\lceil\begin{array}{l}
u_{i} \\
v_{i}
\end{array}\right\rceil=\left\lceil\begin{array}{l}
u_{j} \\
v_{j}
\end{array}\right]+\left[\begin{array}{ll}
\varepsilon_{11} & \varepsilon_{12} \\
\varepsilon_{21} & \varepsilon_{22}
\end{array}\right]\left\lceil\begin{array}{c}
x_{i}-x_{j} \\
y_{i}-y_{j}
\end{array}\right] \text { in } 2 \mathrm{D}
$$

where $u$ and $v$ are the joint displacement field components in the $x$ and $y$ directions, respectively, and joint $i$ is the dependent joint, while joint $j$ is the independent joint. In terms of the engineering strain [23], eqn (22) can be reformulated as:

$$
\left\lceil\begin{array}{l}
u_{i} \\
v_{i}
\end{array}\right\rceil=\left[\begin{array}{l}
u_{j} \\
v_{j}
\end{array}\right]+\left[\begin{array}{cc}
\varepsilon_{11} & \frac{1}{2} \varepsilon_{12} \\
\frac{1}{2} \varepsilon_{21} & \varepsilon_{22}
\end{array}\right]\left\lceil\begin{array}{l}
x_{i}-x_{j} \\
y_{i}-y_{j}
\end{array}\right\rceil \quad \text { in 2D }
$$

which in turn can be expressed as:

$$
\begin{aligned}
& {\left[\begin{array}{l}
u_{i} \\
v_{i}
\end{array}\right]=\left[\begin{array}{l}
u_{j} \\
v_{j}
\end{array}\right]+\left[\begin{array}{ccc}
\left(x_{i}-x_{j}\right) & 0 & \frac{1}{2}\left(y_{i}-y_{j}\right) \\
0 & \left(y_{i}-y_{j}\right) & \frac{1}{2}\left(x_{i}-x_{j}\right)
\end{array}\right]\left[\begin{array}{c}
\varepsilon_{11} \\
\varepsilon_{22} \\
\varepsilon_{21}
\end{array}\right]} \\
& \text { or } d_{i}=d_{j}+\mathrm{E} \varepsilon
\end{aligned}
$$

Equation (24) is the kinematic boundary condition of the Cauchy-Born Hypothesis. Applying this boundary condition to the unit cell joint displacement vector, $d$, results in:

$$
d=T_{d} \tilde{d}+\mathrm{E} \bar{\varepsilon}
$$


Substituting eqn (25) into the kinematic system of the unit cell (eqn (2b)) results in:

$$
B\left\{T_{d} \tilde{d}+\mathrm{E} \bar{\varepsilon}\right\}=e
$$

Substituting eqn (15a) into eqn (26) and inverting $T_{e}$, results in:

$$
\tilde{B} \tilde{d}+\tilde{\mathrm{E}} \bar{\varepsilon}=\tilde{e}
$$

Where $\tilde{B}=\left(B_{e}\right)^{-1} T_{e}^{H} B T_{d}$ and $\tilde{\mathrm{E}}=\left(B_{e}\right)^{-1} T_{e}^{H} B \mathrm{E}$.

From eqns (25) and (26) one can realize that the Cauchy-Born kinematic boundary condition is applied to the kinematic compatibility system of the lattice microstructure to express an explicit relation between the microscopic nodal displacements and a homogeneous averaged macroscopic strain field, $\bar{\varepsilon}$. A key parameter to establish this relation is the existence of the complete nodal periodicity information within the unit cell envelope. The Cauchy-Born hypothesis cannot be applied to the kinematic compatibility relation of the unit cell shown in Fig (1-d) without resorting to the Dummy Node Scheme. This is described by the steps below.

Step 1: Hypothetical dummy nodes are introduced at the intersection points between the microscopic cell elements that extend between neighbouring unit cells, and the cell envelope. These dummy nodes are used to generate the kinematic and the equilibrium matrices of the finite microstructure, as described previously.

Step 2: Equation (7) is applied to the total group of joint bases (including the dummy nodes) to determine the dependent and the independent set of joints.

Step 3: the dependency relations generated in step 2, is now used to apply the CauchyBorn kinematic boundary condition to the kinematic system of the unit cell generated in step 1. This results in a formulation similar to eqn (26). Distributing the bracket in eqn (26), results in:

$$
\underbrace{B T_{d} \tilde{d}}_{1}+\underbrace{B \mathrm{E} \bar{\varepsilon}}_{2}=e
$$

where $B T_{d} \tilde{d} \in R^{\operatorname{dim}(e) \times \operatorname{dim}(\tilde{d})}$ and $B \mathrm{E} \bar{\varepsilon} \in R^{\operatorname{dim}(e) \times 3}$. The first term in eqn (28) left hand side includes the degrees of freedom associated with the dummy nodes.

Step 4: the degrees of freedom associated with the hypothetical dummy nodes, in term (1) of eqn (28), are eliminated from the matrix systems in the same manner as described in section II.1.A.

Step 5: Substituting eqn (15a) into eqn (28) and inverting $T_{e}$, results in: 


$$
\begin{aligned}
& \underbrace{\left(B_{e}\right)^{-1} T_{e}^{H} B T_{d} \tilde{d}}_{1}+\underbrace{\left(B_{e}\right)^{-1} T_{e}^{H} B \mathrm{E} \bar{\varepsilon}}_{2}=\tilde{e} \\
& \text { or } \underbrace{\tilde{\tilde{B}} \tilde{d}}_{1}+\underbrace{\left(B_{e}\right)^{-1} T_{e}^{H} B \mathrm{E} \bar{\varepsilon}}_{2}=\tilde{e}
\end{aligned}
$$

Equation (29) is the complete reduced kinematic system representing the infinite lattice structure.

\section{B. Macroscopic Strain in Terms of Microscopic Element Deformations}

Equation (29) is a matrix system that expresses the periodic element deformations in terms of the macroscopic strain field, $\bar{\varepsilon}$, and the periodic nodal displacements, $\tilde{d}$. This matrix system is rearranged to express the macroscopic strain in terms of the periodic element deformations and as independent of the periodic nodal displacement field, $\tilde{d}$. This is done by generating the following augmented matrix:

$$
[\underbrace{\left(\left(B_{e}\right)^{-1} T_{e}^{H} B T_{d}\right)}_{1}|\underbrace{\left(\left(B_{e}\right)^{-1} T_{e}^{H} B \mathrm{E}\right)}_{2}| \underbrace{(I)}_{3}]
$$

In (30), $I$ is a unit square matrix with dimension equal to $\operatorname{dim}(e)$. The next step is to find the reduced row echelon form of the matrix expressed in (30) and collect the rows in the sub matrices (2) and (3) that correspond to zero rows in the sub matrix (1). This process generates the two matrices $(\tilde{\mathrm{E}})$ and $\tilde{I}$, which are used to write the following expression:

$$
[0] \tilde{d}+(\tilde{\tilde{E}}) \bar{\varepsilon}=\tilde{I} \tilde{e} \quad \text { or }(\tilde{\tilde{E}})-\tilde{\varepsilon}=\tilde{I} \tilde{e}
$$

The matrix system generated in eqn (31) is used to find an explicit expression of the element deformations in terms of the macroscopic strain field. This can be obtained by inverting the matrix $\tilde{I}$. To invert the matrix $\tilde{I}$, we resort to the Moore-Penrose pseudoinverse technique that depends on generating the Singular Value Decomposition [10, 2426] of the matrix $\tilde{I}$ as:

$$
\tilde{I}=S \cdot V \cdot D^{H}
$$

For a $\tilde{I} \in R^{m \times n}$, the singular value decomposition generates the diagonal matrix $V \in R^{m \times n}$, which contains the non-negative Eigenvalues of matrix $\tilde{I}$; the square unitary matrix 
$S \in R^{m \times m}$ and the conjugate transpose matrix $D^{H}$. The Moore-Penrose pseudo-inverse of the matrix $\tilde{I}$, is formulated as:

$$
(\tilde{I})^{-1}=(D)(\tilde{V})^{-1}(S)^{H}
$$

where the term $(\tilde{V})^{-1}$ is formulated by eliminating the rows and the columns of matrix $V$ that have zero diagonal values, and then obtaining the reciprocal of the left diagonal entries. Multiplying eqn (33) to both sides of eqn (31), results in the following expression of the element deformations in terms of the macroscopic strain field:

$$
\tilde{e}=\left((\tilde{I})^{-1}(\tilde{\tilde{E}})\right) \bar{\varepsilon} \quad \text { or } \tilde{e}=M \bar{\varepsilon}
$$

Computing the null space of matrix $M$, gives the independent modes of macroscopic strain field generated with inextensional microscopic element deformations. An empty null space of matrix $M$ indicates that the lattice material can support all macroscopic modes of strain fields. In other words, the material does not fail by periodic mechanisms or any special modes of macroscopic loading.

Finally, the deformations of all elements in the unit cell can be expressed by substituting eqn (34) into eqn (15a) as:

$$
e=T_{e} M \varepsilon
$$

\section{II.4 Macroscopic Strain Energy Density (Material Macroscopic Stiffness Matrix)}

The macroscopic strain energy density of a lattice unit cell with $b$ bars is defined as [2]:

$$
W=\frac{1}{2} \bar{\sigma}: \bar{\varepsilon}=\frac{1}{2|\mathrm{Y}|} \sum_{k=1}^{b} t_{k} e_{k}
$$

where $|\mathrm{Y}|$ is the unit cell area, $t_{k}$ is the tension force in the bar element. $\overline{\bar{\sigma}}$ and $\bar{\varepsilon}$ are the macroscopic stress and strain fields, respectively. Since the lattice structure considered in the current analysis is a pin-jointed structure, then, the bar elements of the unit cell carry only axial loads. Accordingly, the tension force in a bar element, $k$, can be expressed as

$$
t_{k}=(E A / L) e_{k}
$$

where $E$ is the Young's modulus of the solid material, $A$ is the cross-sectional area of the bar element, and $L$ is the bar length. Substituting eqn (37) into eqn (36) results in: 


$$
W=\frac{1}{2} \bar{\sigma}: \bar{\varepsilon}=\frac{E A}{2 L|\mathrm{Y}|} \sum_{k=1}^{b} e_{k}^{2}
$$

Substituting eqn (35) into eqn (38) results in:

$$
W=\frac{1}{2} \bar{\sigma}: \bar{\varepsilon}=\frac{E A}{2 L|\mathrm{Y}|} \sum_{k=1}^{b}(\mathrm{M}(k,:) \bar{\varepsilon})^{2}
$$

where $\mathrm{M}(k,:)$ is the $k^{\text {th }}$ row in the matrix M. Using eqn (39), the macroscopic fourth order stiffness tensor of the lattice material can be computed as:

$$
k_{i i j j}=\frac{\partial^{2} W}{\partial \bar{\varepsilon}_{i i} \partial \overline{\varepsilon_{j j}}}
$$

where $i$ and $j \in\{1, . ., \mathrm{n}\}$ and $\mathrm{n}=2$ or $\mathrm{n}=3$ in $2 \mathrm{D}$ or $3 \mathrm{D}$, respectively.

Once the macroscopic stiffness tensor is computed, the macroscopic compliance matrix can be obtained by inverting the stiffness matrix, where $C_{L}=K_{L}^{-1}$ is the linearly elastic fourth order compliance tensor of the lattice material. For a general anisotropic material the compliance tensor is given by:

$$
\left[\begin{array}{l}
\varepsilon_{x x} \\
\varepsilon_{y y} \\
\varepsilon_{x y}
\end{array}\right]=\left[\begin{array}{lll}
C_{x x x x} & C_{x x y y} & C_{x x x y} \\
C_{y y x x} & C_{y y y y} & C_{y y x y} \\
C_{x y x x} & C_{x y y y} & C_{x y x y}
\end{array}\right]\left[\begin{array}{l}
\sigma_{x x} \\
\sigma_{y y} \\
\sigma_{x y}
\end{array}\right] \quad \text { or } \bar{\varepsilon}=C_{L} \bar{\sigma}
$$

The compliance tensor can be used to compute the lattice material elastic moduli as:

$$
\begin{aligned}
& \left(E_{L}\right)_{x x}=\frac{1}{C_{x x x x}} \\
& \left(E_{L}\right)_{y y}=\frac{1}{C_{y y y y}} \\
& \left(v_{L}\right)_{y x}=-\frac{C_{x x x x}}{C_{x x y y}} \\
& \left(v_{L}\right)_{x y}=-\frac{C_{y y y y}}{C_{y y x x}} \\
& G_{L}=\frac{1}{C_{x y x y}}
\end{aligned}
$$


Where $\left(E_{L}\right)_{i j}$ and $\left(v_{L}\right)_{i j}$ are, respectively, the material Young's modulus and Poisson's ratio in the $i j$ direction, and $i, j \in\{x, y\}$, and $G$ is the shear modulus of the material.

\section{CHARACTERIZATION OF 2D LATTICE MATERIALS III.1 Lattice Materials with Hexagonal Bravais Lattice Symmetry}

In this study, we consider two cell topologies (Fig (4)) with hexagonal Bravais lattice symmetry that so far have not been characterized in literature. The hexagonal symmetry is illustrated by the cell envelope in each lattice. Lattice materials with hexagonal Bravais lattice symmetry available in literature [2- 4] are shown in Fig (5). We use the method described in the previous sections to determine the elastostatic stiffness properties of the lattice materials shown in Figs (4) and (5).

\section{A. $3^{4} .6$ Lattice Material}

\section{A.1 Analysis of Unit Cell Finite Structure}

The unit cell of the $3^{4} .6$ lattice contains 6 real joints and 24 bars, as shown in Fig (4). Since there are 18 intersection points between the cell envelope and the bar elements that extend between adjacent cells, we introduce a dummy node for each intersection. The groups of bar and joint position vectors are used to formulate the kinematic and equilibrium matrices of the unit cell structure.

The determinacy analysis of the unit cell structure reveals that the cell is statically determinate since it does not include any states of self-stress; however, 21 internal mechanisms make it kinematically indeterminate.

\section{A.2 Determinacy Analysis of Infinite Structure}

(1) The Direct Lattice

From the geometry of the unit cell envelope, the direct translational bases can be formulated as $\vec{a}_{1}=-2.5 \hat{i}+0.866 \hat{j}, \vec{a}_{2}=-2 \hat{i}+1.7321 \hat{j}$, where $\hat{i}$ and $\hat{j}$ are the $2 \mathrm{D}$ Cartesian space unit vectors. To determine the direct lattice bases, the dependency between the unit cell bar and joint position vectors is verified on a unit cell bases using (eqn (7)). This test reveals that all the joints are independent whereas the bars exhibit dependencies, as shown in Table (1).

\section{Table (1) Unit Cell Bars Dependency Relations}

\section{Fig (4) New Cell Topologies with Hexagonal Bravais Lattice Symmetry}

\section{Fig (5) Cell Topologies with Hexagonal Bravais Lattice Symmetry available in the literature}

The numeric tags of the cell elements (table (1)) are used in Fig (4) to label the elements of the unit cell of the $3^{4} .6$ lattice. The dependency relations are used to generate the bars and the joints transformation matrices, which are necessary to reduce the kinematic and the equilibrium systems to their periodic forms.

\section{(2) The Reciprocal Lattice}


After the reciprocal lattice and the first Brillouin Zone [1] are determined, point group symmetry [27-29] is used to determine the irreducible first Brillouin Zone which is used to generate the critical k-points (wave vectors), as illustrated in Fig (6). The values of the critical k-points are shown in Table (2).

Fig (6) First Brillouin Zone and Irreducible Brillouin Zone of the $3^{4} .6$ Lattice

\section{Table (2) The critical k-points in the Irreducible Brillouin Zone}

The reduced equilibrium and kinematic matrices are computed at each critical k-point vector and the determinacy state of the infinite structure is computed. The determinacy analysis shows that the infinite structure of the $3^{4} .6$ lattice is always kinematically determinate and statically indeterminate.

\section{(3) Macroscopic Strain Generated by Microscopic Mechanisms (Cauchy Born Hypothesis)}

Since the infinite structure does not contain any microscopic mechanisms, then it is known that there are no periodic mechanism failure modes. However, the analysis using the Cauchy-Born hypothesis is carried out to verify that no special macroscopic strain fields at which the lattice looses stiffness are present. As explained previously, the Dummy Node Scheme is used to generate the matrix E, which is necessary to formulate the kinematic boundary condition of the Cauchy Born Hypothesis. The singular value decomposition is used to formulate the microscopic element deformations in terms of the macroscopic strain field through the transformation matrix, $M$. The null space of the matrix $M$ is finally computed to identify any special failure modes of macroscopic strain fields. The analysis shows that the $3^{4} .6$ lattice is stable under all macroscopic strain fields.

\section{(4) Macroscopic Stiffness}

The element deformations in (3) are used to determine the strain energy density (eqn (39)) and then to compute the macroscopic stiffness (eqn (40)) of the lattice. Finally, the compliance matrix of the material and the material elastic moduli (eqn (42)) can be derived. For a lattice material with a unit out of plane thickness, the stiffness and the density are written as:

$$
\begin{gathered}
K_{L}=\frac{E H}{L}\left[\begin{array}{ccc}
1.0998 & 0.5976 & 0 \\
0.5976 & 1.0998 & 0 \\
0 & 0 & 0.2511
\end{array}\right]=E \bar{\rho}_{L}\left[\begin{array}{ccc}
0.4445 & 0.2415 & 0 \\
0.2415 & 0.4445 & 0 \\
0 & 0 & 0.1015
\end{array}\right], \\
\bar{K}_{L}=\frac{K_{L}}{E}=\bar{\rho}_{L}\left[\begin{array}{ccc}
0.4445 & 0.2415 & 0 \\
0.2415 & 0.4445 & 0 \\
0 & 0 & 0.1015
\end{array}\right], \bar{\rho}_{L}=2.4744\left(\frac{H}{L}\right)
\end{gathered}
$$

where $\bar{\rho}_{L}, K_{L}$ and $\bar{K}_{L}$ are the lattice material relative density, stiffness matrix and relative stiffness matrix, respectively. While $E$ and $H$ are the solid material Young's modulus and cell element in the plane thickness, respectively. 
Once the stiffness tensor is computed, the compliance tensor can be computed as:

$$
C_{L}=\frac{1}{E \bar{\rho}_{L}}\left[\begin{array}{ccc}
3.1919 & -1.7342 & 0 \\
-1.7342 & 3.1919 & 0 \\
0 & 0 & 9.8522
\end{array}\right]
$$

This compliance tensor is used to compute the material elastic moduli as:

$$
\left(\bar{E}_{L}\right)_{x x}=\frac{\left(E_{L}\right)_{x x}}{E}=0.3133 \bar{\rho}_{L},\left(\bar{E}_{L}\right)_{y y}=\frac{\left(E_{L}\right)_{y y}}{E}=0.3133 \bar{\rho}_{L}, \bar{G}_{L}=\frac{G_{L}}{E}=0.1015 \bar{\rho}_{L}
$$

The same analysis is carried out for the other lattices shown in Figs (4) and (5). The final results are shown below.

\section{A. Double Hexagonal Triangulation (DHT)}

$$
\begin{gathered}
K_{L}=\frac{E H}{L}\left[\begin{array}{ccc}
0.9575 & 0.388 & 0 \\
0.388 & 0.9575 & 0 \\
0 & 0 & 0.2595
\end{array}\right]=E \rho_{L}\left[\begin{array}{ccc}
0.3431 & 0.1391 & 0 \\
0.1391 & 0.3431 & 0 \\
0 & 0 & 0.093
\end{array}\right] \\
\bar{K}_{L}=\frac{K_{L}}{E}=\bar{\rho}_{L}\left[\begin{array}{ccc}
0.3431 & 0.1391 & 0 \\
0.1391 & 0.3431 & 0 \\
0 & 0 & 0.093
\end{array}\right], \bar{\rho}_{L}=2.7905\left(\frac{H}{L}\right) \\
\left(\bar{E}_{L}\right)_{x x}=\frac{\left(E_{L}\right)_{x x}}{E}=0.2659 \bar{\rho}_{L},\left(\bar{E}_{L}\right)_{y y}=\frac{\left(E_{L}\right)_{y y}}{E}=0.1972 \bar{\rho}_{L}, \bar{G}_{L}=\frac{G_{L}}{E}=0.0878 \rho_{L}
\end{gathered}
$$

\section{B. Full Triangulation $\left(3^{6}\right)$}

$$
\begin{array}{r}
K_{L}=\frac{E H}{L}\left[\begin{array}{ccc}
1.299 & 0.433 & 0 \\
0.433 & 1.299 & 0 \\
0 & 0 & 0.433
\end{array}\right]=E \bar{\rho}_{L}\left[\begin{array}{ccc}
0.375 & 0.125 & 0 \\
0.125 & 0.375 & 0 \\
0 & 0 & 0.125
\end{array}\right], \\
\bar{K}_{L}=\frac{K_{L}}{E}=\bar{\rho}_{L}\left[\begin{array}{ccc}
0.375 & 0.125 & 0 \\
0.125 & 0.375 & 0 \\
0 & 0 & 0.125
\end{array}\right], \bar{\rho}_{L}=3.4641\left(\frac{H}{L}\right) \\
\left(\bar{E}_{L}\right)_{x x}=\frac{\left(E_{L}\right)_{x x}}{E}=0.3333 \bar{\rho}_{L},\left(\bar{E}_{L}\right)_{y y}=\frac{\left(E_{L}\right)_{y y}}{E}=0.3333 \bar{\rho}_{L}, \bar{G}_{L}=\frac{G_{L}}{E}=0.125 \bar{\rho}_{L}
\end{array}
$$

\section{Hexagonal Honeycombs}




$$
\begin{gathered}
K_{L}=\frac{E H}{L}\left[\begin{array}{ccc}
0.2887 & 0.2887 & 0 \\
0.2887 & 0.2887 & 0 \\
0 & 0 & 0
\end{array}\right]=E \bar{\rho}_{L}\left[\begin{array}{ccc}
0.25 & 0.25 & 0 \\
0.25 & 0.25 & 0 \\
0 & 0 & 0
\end{array}\right] \\
\bar{K}_{L}=\frac{K_{L}}{E}=\bar{\rho}_{L}\left[\begin{array}{ccc}
0.25 & 0.25 & 0 \\
0.25 & 0.25 & 0 \\
0 & 0 & 0
\end{array}\right], \bar{\rho}_{L}=1.1547\left(\frac{H}{L}\right)
\end{gathered}
$$

Since this lattice structure is bending-dominated, the stiffness matrix of its pin jointed lattice version is singular. Therefore, the compliance matrix and elastic moduli loose their significance. We do not present them here, as this paper focuses on stretching-dominated lattice material and the modeling of rigid-jointed lattice is out of the scope.

\section{Kagome'}

$$
\begin{gathered}
K_{L}=\frac{E H}{L}\left[\begin{array}{ccc}
0.6495 & 0.2165 & 0 \\
0.2165 & 0.6495 & 0 \\
0 & 0 & 0.2165
\end{array}\right]=E \bar{\rho}_{L}\left[\begin{array}{ccc}
0.375 & 0.125 & 0 \\
0.125 & 0.375 & 0 \\
0 & 0 & 0.125
\end{array}\right], \\
\bar{K}_{L}=\frac{K_{L}}{E}=\bar{\rho}_{L}\left[\begin{array}{ccc}
0.375 & 0.125 & 0 \\
0.125 & 0.375 & 0 \\
0 & 0 & 0.125
\end{array}\right], \bar{\rho}_{L}=1.7321\left(\frac{H}{L}\right) \\
\left(\bar{E}_{L}\right)_{x x}=\frac{\left(E_{L}\right)_{x x}}{E}=0.3333 \bar{\rho}_{L},\left(\bar{E}_{L}\right)_{y y}=\frac{\left(E_{L}\right)_{y y}}{E}=0.3333 \bar{\rho}_{L}, \bar{G}_{L}=\frac{G_{L}}{E}=0.125 \rho_{L}
\end{gathered}
$$

\section{III.2 Lattice Materials with Square Bravais Lattice Symmetry}

\section{A. Determinacy Analysis}

Figure (7) shows five lattice topologies with square Bravais lattice symmetry examined in this paper. For these topologies, we follow the previous procedure; our goal is to determine stiffness properties and static performance, i.e. specific stiffness.

The determinacy analysis of the finite structures of the unit cells of the five lattice topologies reveals that unit cell (a) is kinematically indeterminate and statically determinate; on the other hand, unit cells (b), (c), (d) and (e) are kinematically and statically determinate. Extending the analysis to the infinite lattice structures using the Bloch's theorem, reveals that lattices (a) and (b) are kinematically and statically indeterminate; on the other hand, lattices (c), (d) and (e) are kinematically determinate and statically indeterminate.

\section{Fig (7) Cell Topologies with Square Bravais Lattice Symmetry}

Using the Cauchy-Born hypothesis, we search macroscopic strain fields generated by inextensional microscopic mechanisms; we find that lattice (a) has one shear macroscopic strain field associated with the inextensional microscopic mechanisms. On the contrary, lattice (b) does not have any macroscopic strain field associated with microscopic mechanisms, for its microscopic mechanism vanishes on the macro scale. For lattices (c), 
(d) and (e) there are no failure macroscopic strain fields. This analysis indicates that lattice (a) is a bending-dominated lattice material and lattices (b), (c), (d) and (e) are stretching-dominated lattice materials.

\section{B. Stiffness Properties}

The strain energy density is formulated and used to derive the averaged stiffness properties of the five lattices shown in Fig (7) as:

- Lattice (a)

$$
\bar{K}_{L}=\frac{K_{L}}{E}=\bar{\rho}_{L}\left[\begin{array}{ccc}
0.5 & 0 & 0 \\
0 & 0.5 & 0 \\
0 & 0 & 0
\end{array}\right], \bar{\rho}_{L}=2\left(\frac{H}{L}\right)
$$

The computation of the compliance matrix and the elastic moduli are not presented as the material is bending-dominated.

- $\quad$ Lattice (b)

$$
\begin{array}{r}
\bar{K}_{L}=\frac{K_{L}}{E}=\bar{\rho}_{L}\left[\begin{array}{ccc}
0.4347 & 0.0653 & -0.0653 \\
0.0653 & 0.4347 & -0.0653 \\
-0.0653 & -0.0653 & 0.0653
\end{array}\right], \bar{\rho}_{L}=2.71\left(\frac{H}{L}\right) \\
\left(\bar{E}_{L}\right)_{x x}=\frac{\left(E_{L}\right)_{x x}}{E}=0.3694 \bar{\rho}_{L},\left(\bar{E}_{L}\right)_{y y}=\frac{\left(E_{L}\right)_{y y}}{E}=0.3694 \bar{\rho}_{L}, \bar{G}_{L}=\frac{G_{L}}{E}=0.0482 \bar{\rho}_{L}
\end{array}
$$

- $\quad$ Lattice (c)

$$
\begin{gathered}
\bar{K}_{L}=\frac{K_{L}}{E}=\bar{\rho}_{L}\left[\begin{array}{ccc}
0.3964 & 0.1036 & 0 \\
0.1036 & 0.3964 & 0 \\
0 & 0 & 0.1036
\end{array}\right], \bar{\rho}_{L}=3.41\left(\frac{H}{L}\right) \\
\left(\bar{E}_{L}\right)_{x x}=\frac{\left(E_{L}\right)_{x x}}{E}=0.3693 \bar{\rho}_{L},\left(\bar{E}_{L}\right)_{y y}=\frac{\left(E_{L}\right)_{y y}}{E}=0.3693 \bar{\rho}_{L}, \bar{G}_{L}=\frac{G_{L}}{E}=0.1036 \bar{\rho}_{L}
\end{gathered}
$$

- $\quad$ Lattice $(d)$

$$
\begin{array}{r}
\bar{K}_{L}=\frac{K_{L}}{E}=\bar{\rho}_{L}\left[\begin{array}{ccc}
0.3964 & 0.1036 & -0.1036 \\
0.1036 & 0.3964 & -0.1036 \\
-0.1036 & -0.1036 & 0.1036
\end{array}\right], \bar{\rho}_{L}=3.4142\left(\frac{H}{L}\right) \\
\left(\bar{E}_{L}\right)_{x x}=\frac{\left(E_{L}\right)_{x x}}{E}=0.2928 \bar{\rho}_{L},\left(\bar{E}_{L}\right)_{y y}=\frac{\left(E_{L}\right)_{y y}}{E}=0.2928 \bar{\rho}_{L}, \bar{G}_{L}=\frac{G_{L}}{E}=0.0607 \bar{\rho}_{L}
\end{array}
$$

- $\quad$ Lattice (e)

$$
\bar{K}_{L}=\frac{K_{L}}{E}=\bar{\rho}_{L}\left[\begin{array}{ccc}
0.3536 & 0.1464 & 0 \\
0.1464 & 0.3536 & 0 \\
0 & 0 & 0.1464
\end{array}\right], \bar{\rho}_{L}=4.8284\left(\frac{H}{L}\right)
$$


$\left(\bar{E}_{L}\right)_{x x}=\frac{\left(E_{L}\right)_{x x}}{E}=0.293 \bar{\rho}_{L},\left(\bar{E}_{L}\right)_{y y}=\frac{\left(E_{L}\right)_{y y}}{E}=0.293 \bar{\rho}_{L}, \bar{G}_{L}=\frac{G_{L}}{E}=0.1464 \bar{\rho}_{L}$

\section{CONCLUDING REMARKS}

This paper has described a systematic matrix-based procedure for the specific stiffness characterization of lattice materials with any arbitrary topology. This procedure is efficient for the automation of the characterization process of complex microscopic topologies. A scheme based on the concept of dummy nodes has been introduced to deal with lattice materials that consist of unit cell elements intersecting their cell envelopes. The procedure has been applied to lattice materials with hexagonal and square Bravais symmetries. The results have been plotted on design charts that can help in the selection process of lattice topologies for given stiffness requirements. It is found that the lattice materials with cell topologies shown in Fig (7-b) and (7-c) exhibit $11 \%$ increase of the specific stiffness compared to the Kagome' and the full triangulation lattice materials. On the other hand, the lattice material with cell topology shown in Fig (7-e) shows $17 \%$ improvement in the specific shear modulus compared to the Kagome' and the full triangulation lattice materials.

Fig (8) Relative Young's modulus in the $x$ direction (see Figs. 4,5, 7) versus relative density of selected 2D lattice materials

Fig (9) Relative Young's modulus in the y direction (see Figs. 4,5, 7) versus relative density of selected 2D lattice materials

\section{Fig (10) Relative Shear modulus versus relative density of selected 2D lattice materials}

\section{Appendix A: The dummy Node Scheme}

Consider the sequence of three unit cells describing the periodicity of the lattice structure shown in Fig (11). We specify two elements, $a$ and $b$, of lengths $L_{a}$ and $L_{b}$. Element $a$ is connected between node $n 1$, located on the borders between unit cells I and II, and node $n 2$ that belongs to unit cell III. Element $b$ is connected between node $n 3$, belongs to unit cell I, and node $n 4$ which belongs to unit cell II.

Fig (11) Lattice structure (left) and zoom on three unit cells (right) tessellated in the direction of the horizontal translational basis.

Legend: Continuous lines: structural elements; Dashed lines: cell envelopes; O: Real structural nodes; $\mathbf{0}$ : Dummy nodes

The envelope of unit cell II intersects elements $a$ and $b$ respectively at nodes $n 5$ and $n 6$, which are dummy nodes introduced at intersection points between envelope and unit cell elements. Node $n 5$ splits element $a$ into two segments $a 1$ and $a 2$ of length $L_{a 1}$ and $L_{a 2}$, respectively. On the other hand, node $n 6$ divides element $b$ into two segments $b 1$ and $b 2$ of length $L_{b 1}$ and $L_{b 2}$, respectively. Elements $a$ and $b$ carry internal tension forces $t_{a}$ and $t_{b}$, respectively. Thus, the portions of the nodal forces that are in balance with the tension 
forces in elements $a$ and $b$ are specified by a two dimensional vector $\mathbf{f}_{n i}$ that has two components in the $x$ and the $y$ directions of the Cartesian coordinates. If $\vec{r}_{n i}$ is the position vector of node $n i$ and $i \in\{1,2,3,4,5,6\}$, then a unit vector in the direction of elements $\mathrm{a}$ and $\mathrm{b}$ can be written as:

$$
\begin{aligned}
& \hat{n}_{a}=\frac{\vec{r}_{n 5}-\vec{r}_{n 1}}{L_{a 1}}=\frac{\vec{r}_{n 2}-\vec{r}_{n 5}}{L_{a 2}}=\frac{\vec{r}_{n 2}-\vec{r}_{n 1}}{L_{a}} \\
& \hat{n}_{b}=\frac{\vec{r}_{n 6}-\vec{r}_{n 3}}{L_{b 1}}=\frac{\vec{r}_{n 4}-\vec{r}_{n 6}}{L_{b 2}}=\frac{\vec{r}_{n 4}-\vec{r}_{n 3}}{L_{b}}
\end{aligned}
$$

\section{A.1 Equilibrium Analysis}

The static equilibrium system of a structure that has $b$ elements connected between $j$ nodes is represented as:

$$
A t=f
$$

where $A \in R^{n j \times b}, n=2$ in $2 \mathrm{D}$, is a Jacobian matrix with entries of direction cosines that transforms the vector of tension forces of the structural elements $t \in R^{b}$ to the vector of the nodal forces $f \in R^{n j}[30,31]$.

Consider the segment $a 1$ of element $a$; the static equilibrium of forces at nodes $n 1$ and $n 5$ with the tension force in the element $t_{a}$ can be written as:

$$
\left\lceil\begin{array}{c}
-\hat{n}_{a} \\
\hat{n}_{a}
\end{array}\right] t_{a}=\left\lceil\begin{array}{c}
f_{n 1} \\
f_{n 5}
\end{array}\right]
$$

Similarly, consider the segment $a 2$ of element $a$, the static equilibrium of forces at nodes $n 5$ and $n 2$ with the tension force in the element $t_{a}$ is given by:

$$
\left\lceil\begin{array}{c}
-\hat{n}_{a} \\
\hat{n}_{a}
\end{array}\right] t_{a}=\left\lceil\begin{array}{c}
f_{n 5} \\
f_{n 2}
\end{array}\right\rceil
$$

The assembly of eqns (A4) and (A5) into one matrix system results in:

$$
\left\lceil\begin{array}{c}
\hat{-n_{a}} \\
\hat{n}_{a}-\hat{n}_{a} \\
\hat{n}_{a}
\end{array}\right] t_{a}=\left[\begin{array}{c}
-\hat{n}_{a} \\
0 \\
\hat{n_{a}}
\end{array}\right] t_{a}=\left[\begin{array}{c}
f_{n 1} \\
f_{n 5} \\
f_{n 2}
\end{array}\right]
$$


From eqn (A6) one can realize that the coefficients of the dummy node, $n 5$, can be set to zero to eliminate the node from the matrix system, which results in:

$$
\left\lceil\begin{array}{c}
\hat{-} n_{a} \\
n_{a}
\end{array}\right] t_{a}=\left\lceil\begin{array}{c}
f_{n 1} \\
f_{n 2}
\end{array}\right]
$$

The same reasoning can be applied to element $b$, where the equilibrium of the nodal forces at nodes $n 3, n 4$ and $n 6$ with the element tension force $t_{b}$ can be expressed respectively in eqns (A8) and (A9) as:

$$
\begin{aligned}
& {\left[\begin{array}{c}
\hat{-} \\
\hat{n_{b}} \\
n_{b}
\end{array}\right] t_{b}=\left\lceil\begin{array}{c}
f_{n 3} \\
f_{n 6}
\end{array}\right]} \\
& {\left[\begin{array}{c}
\hat{\wedge} \\
-n_{b} \\
n_{b}
\end{array}\right] t_{b}=\left[\begin{array}{c}
f_{n 6} \\
f_{n 4}
\end{array}\right]}
\end{aligned}
$$

The assembly of eqns (A8) and (A9) in one matrix system results in:

$$
\left[\begin{array}{c}
\hat{-} \\
\hat{n} \\
n_{b}
\end{array}\right] t_{b}=\left\lceil\begin{array}{c}
f_{n 3} \\
f_{n 4}
\end{array}\right]
$$

Now, we consider only the equilibrium of the portions of elements $a$ and $b$ enclosed within the envelope of cell II. Those are the segment $a 1$ of element $a$ and the segment $b 2$ of element $b$. It is noted that the existence of a dummy node $n d 1$ implies always the existence of a dummy node $n d 2$ where node $n d 1$ is dependent on node $n d 2$, or vice versa, as a result of the translational symmetry. If we assume that a pair of dummy nodes consists of nodes $n d 1$ and $n d 2$, then a number of properties can be identified.

1) The dependency relationship between nodes $n d 1$ and $n d 2$ can be expressed through their position vectors $\vec{r}_{n d 1}$ and $\vec{r}_{n d 2}$ as:

$$
\vec{r}_{n d 1}=\vec{r}_{n d 2} \pm \vec{a}_{k}
$$

where $k \in\{1,2\}$ in $2 \mathrm{D}$.

2) Within the unit cell envelope, if node $n d 1$ is located on element $e 1$ and node $n d 2$ is located on element $e 2$, then element $e 1$ is the complementary of element $e 2$ for the portion of $e 2$ that is not included within the cell envelope. Similarly, $e 2$ is the complementary of element $e 1$ for the portion of $e 1$ that is not included within the cell envelope. Also, the static wave functions, associated with nodes $n d 1$ and $n d 2$ as well as those associated with elements $e 1$ and $e 2$, follow the anti-periodic constraints, necessary 
for the static equilibrium of the lattice. These properties are expressed by the following static conditions: direction.

a) Tension forces of elements $e 1$ and $e 2$ are equal in magnitude and opposite in

b) Nodal forces at nodes $n d 1$ and $n d 2$ are equal in magnitude and opposite in direction.

In addition to the geometrical condition that:

c) Unit vectors in the directions of elements $e 1$ and $e 2$ are parallel.

Fig (1) shows that nodes $n 5$ and $n 6$ are two dependent dummy; thus they must satisfy the following properties:

$$
\hat{n}_{a}=\hat{n}_{b}, t_{a}=-t_{b} \text { and } f_{n 5}=-f_{n 6}
$$

Now, consider the equilibrium of portion $a 1$ of element $a$, formulated in eqn (A4), and the equilibrium of portion $b 2$ of element $b$ that is formulated in eqn (A9). The assembly of eqns (A4) and (A9) in one matrix system results in:

$$
\left[\begin{array}{cc}
\wedge & \\
-n_{a} & 0 \\
\hat{n}_{a} & 0 \\
0 & -\hat{n}_{b} \\
0 & n_{b}
\end{array}\right]\left[\begin{array}{l}
t_{a} \\
t_{b}
\end{array}\right]=\left[\begin{array}{c}
f_{n 1} \\
f_{n 5} \\
f_{n 6} \\
f_{n 4}
\end{array}\right]
$$

Applying the conditions of eqn (A12), results in:

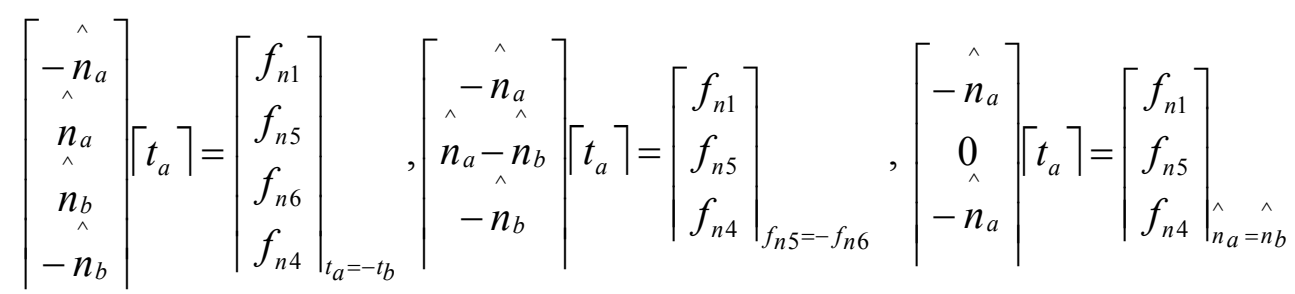

where the subscript denotes the applied condition. Finally the matrix system of eqn (A14) is reduced to:

$$
\left\lceil\begin{array}{c}
\hat{n_{a}} \\
-\hat{n}_{a}
\end{array}\right] t_{a}=\left[\begin{array}{cc}
\hat{n_{a}} & 0 \\
0 & \hat{n_{a}}
\end{array}\right]\left[\begin{array}{l}
t_{a} \\
t_{b}
\end{array}\right]=\left\lceil\begin{array}{c}
f_{n 1} \\
f_{n 4}
\end{array}\right]
$$

which is equivalent to the elimination of the degrees of freedom of dummy nodes $n 5$ and $n 6$ from the matrix system. It should be noted that the matrix system obtained in eqn (A15) is identical to the results obtained in eqn (A7) and eqn (A10). 


\section{A.2 Kinematic Analysis}

Similar to the Equilibrium Analysis, the kinematic system of a structure that has $b$ elements connected between $j$ nodes is represented as:

$$
B d=e
$$

where $B \in R^{b \times n j}, n=2$ in $2 \mathrm{D}$, is a Jacobian matrix of entries of direction cosines that transforms the vector of nodal displacements $d \in R^{n j}$ to the vector of element deformations $e \in R^{b}[30,31]$.

Consider the segment $a 1$ of element $a$, the kinematic compatibility of displacements of nodes $n 1$ and $n 5$ with the deformation of the element portion, $e_{a 1}$ can be written as:

$$
\left[\begin{array}{cc}
\hat{n}_{a} & -\hat{n}_{a}
\end{array}\right]\left[\begin{array}{l}
d_{n 1} \\
d_{n 5}
\end{array}\right]=e_{a 1}
$$

Similarly, consider the segment $a 2$ of element $a$, the kinematic compatibility of displacements of nodes $n 5$ and $n 2$ with the deformation of the element portion, $e_{a 2}$ can be written as:

$$
\left[\begin{array}{cc}
\hat{n}_{a} & -\hat{n}_{a}
\end{array}\right]\left[\begin{array}{l}
d_{n 5} \\
d_{n 2}
\end{array}\right]=e_{a 2}
$$

The assembly of eqns (A17) and (A18) into one matrix system results in:

$$
\left[\begin{array}{lll}
\hat{n}_{a} & \hat{n}_{a}-\hat{n}_{a} & -\hat{n}_{a}
\end{array}\right]\left[\begin{array}{l}
d_{n 1} \\
d_{n 5} \\
d_{n 2}
\end{array}\right]=\left[\begin{array}{lll}
\hat{n}_{a} & 0 & -\hat{n}_{a}
\end{array}\right]\left[\begin{array}{c}
d_{n 1} \\
d_{n 5} \\
d_{n 2}
\end{array}\right]=e_{a 1}+e_{a 2}=e_{a}
$$

From eqn (A19) one can realize that the coefficients of the dummy node, $n 5$, can be set to zero to eliminate the node from the matrix system, which results in:

$$
\left[\begin{array}{cc}
\hat{n}_{a} & -\hat{n}_{a}
\end{array}\right]\left[\begin{array}{c}
d_{n 1} \\
d_{n 2}
\end{array}\right]=e_{a}
$$

The same reasoning can be applied to element $b$, where the kinematic compatibility of the displacements of nodes $n 3, n 4$ and $n 6$ with the element deformation, $e_{b}$ can be expressed respectively in eqns (A21) and (A22) as: 


$$
\begin{gathered}
{\left[\begin{array}{ll}
\hat{n}_{b} & -\hat{n}_{b}
\end{array}\right]\left[\begin{array}{l}
d_{n 3} \\
d_{n 6}
\end{array}\right]=e_{b 1}} \\
{\left[\begin{array}{ll}
\hat{n}_{b} & -\hat{n}_{b}
\end{array}\right]\left[\begin{array}{l}
d_{n 6} \\
d_{n 4}
\end{array}\right]=e_{b 2}}
\end{gathered}
$$

The assembly of eqns (A21) and (A22) in one matrix system results in:

$$
\left[\begin{array}{cc}
n_{b} & -\hat{n}_{b}
\end{array}\right]\left[\begin{array}{l}
d_{n 3} \\
d_{n 4}
\end{array}\right]=e_{b}
$$

Now, we consider only the kinematic compatibility of the portions of elements $a$ and $b$ enclosed within the envelop of cell II. Those are segment $a 1$ of element $a$ and segment $b 2$ of element $b$.

Also in this case, due to the translational symmetry of lattice structures, if nodes $n d 1$ and $n d 2$ are two dependent dummy nodes, and if node $n d 1$ is located on element $e 1$ and node $n d 2$ is located on element $e 2$, then element $e 1$ is the complementary of element $e 2$ for the portion of $e 2$ that is not included within the cell envelope; and vice versa, $e 2$ is the complementary of element $e 1$ for the portion of $e 1$ that is not included within the cell envelope. This feature imposes the following compatibility conditions:

a) The total deformation of element $e 1$ (total deformation is equal to the deformation of the portion included within the cell envelope and the portion external to the cell envelope) and the total deformation of element $e 2$ are equal.

b) The summation of the deformations of the two element parts enclosed within the cell envelope is equal to the total deformation of element $e 1$ or $e 2$.

c) The displacement of nodes $n d 1$ and $n d 2$ are equal.

From Fig (11), it can be realized that nodes $n 5$ and $n 6$ are two dependent dummy nodes, i.e. they impose the following kinematic properties:

$$
e_{a 1}+e_{b 2}=e_{a 2}+e_{b 1}=e_{a}=e_{b} \text { and } d_{n 5}=d_{n 6}
$$

Now, consider the kinematic compatibility of segment $a 1$ of element $a$, formulated in eqn (A17), and the kinematic compatibility of segment $b 2$ of element $b$ that is formulated in eqn (A22). The assembly of eqns (A17) and (A22) in one matrix system results in:

$$
\left[\begin{array}{llll}
\hat{n}_{a} & -\hat{n}_{a} & \hat{n}_{b} & -\hat{n}_{b}
\end{array}\right]\left[\begin{array}{l}
d_{n 1} \\
d_{n 5} \\
d_{n 6} \\
d_{n 4}
\end{array}\right]=e_{a 1}+e_{b 2}
$$

Applying the conditions of eqn (A24), results in: 


$$
\left[\begin{array}{ccc}
\hat{n_{a}} & \hat{n}_{b}-\hat{n}_{a} & -\hat{n}_{b}
\end{array}\right]\left[\begin{array}{l}
d_{n 1} \\
d_{n 5} \\
d_{n 4}
\end{array}\right]_{d_{n 5}=d_{n 6}}=\left[\begin{array}{lll}
\hat{n}_{a} & 0 & -\hat{n}_{b}
\end{array}\right]\left[\left.\begin{array}{l}
d_{n 1} \\
d_{n 5} \\
d_{n 4}
\end{array}\right|_{\hat{n}_{a}=n_{b}}=e_{a}\right.
$$

where the subscript denotes the applied condition. Finally the matrix system of eqn (A26) is reduced to:

$$
\left[\begin{array}{cc}
\hat{n} & -\hat{n}_{b}
\end{array}\right]\left[\begin{array}{l}
d_{n 1} \\
d_{n 4}
\end{array}\right]=e_{a}
$$

which is equivalent to the elimination of the degrees of freedom of nodes $n 5$ and $n 6$, i.e. the dummy nodes, from the matrix system.

From this analysis, it is noteworthy that the Dummy Node Scheme is an adequate technique to simplify the matrix computation of lattice structures. Since in the kinematic determinacy analysis the lattice structure is considered as pin-jointed, failure of eliminating the degrees of freedom associated with the dummy nodes results in inaccurate results. Furthermore, the above analysis is concerned with the derivation of the kinematic and the equilibrium systems of the unit cell finite structure, which implies that the translational periodicity applied to the unit cell envelope is formulated at the wave number $\omega=(0,0)$, as defined by the Bloch's theorem.

\section{Appendix B Transformation Matrices}

The procedure to obtain the transformation matrix, $T$, for a set of vectors, $V \in R^{n}$, depends on the dependency relation of the vectors. If all vectors are independent, then $V$ can be written as:

$$
V=I V
$$

where $I \in R^{n \times n}$ is a unit square matrix.

On the other hand, if some vectors are dependent vectors, then Equation (B1) can be modified as:

$$
V=T V_{\text {ind }}
$$

where $V_{\text {ind }}$ is the set of independent vectors. Here, $T$ is a modification of $I$.

If we assume that the vector with order $n_{1}$ of the set, $V$, is dependent on the vector with order $n_{2}$, then we can modify $I$ to generate $T$; the column number $n_{1}$ in $I$ is to be eliminated and the element with index $T\left(n_{1}, n_{2}\right)$ modified to:

$$
T\left(n_{1}, n_{2}\right)=e^{2 \pi\left(\hat{\omega_{1} x_{1}+\omega_{2} x_{2}}\right) i}
$$


where $\hat{x}_{1}, x_{2} \in\{1,0,-1\}$ with respect to the vectors dependency relation expressed by eqn (7). This process requires that the dependent vectors are arranged in a descending order during the column elimination process to avoid column order swap.

\section{REFERENCES}

[1] Brillouin, L., International series in pure and applied physics. New York: McGrawHill, 1946.

[2] Hutchinson, R. G. \& Fleck, N.A., Journal of the Mechanics and Physics of Solids, 54, 4, 756-782, 2006.

[3] Phani, S.A., Woodhouse, J., \& Fleck, N. A., Journal of the Acoustical Society of America, 119, 4, 1995-2005, 2006.

[4] Hutchinson R.G., Ph.D. thesis, Cambridge University, 2004.

[5] Alethea M. H., (2004), J. Mechanics of Materials, 36, 691-713.

[6] Gibson L.J. \& Ashby M.F., 2nd ed., Cambridge University Press, 1997.

[7] Maxwell, J. C., Philosophical Magazine, 27, 294, 1864, paper XXVI in Collected papers, Cambridge 1890.

[8] Calladine, C. R., Int. J. Solids. Structures, 14, 161- 172, 1978.

[9] Pellegrino, S., \& Calladine, C. R., Int. J. Solids. Structures, 22, 4, 409- 428, 1986.

[10] Pellegrino, S., Int. J. Solids. Structures, 34, 21, 3025- 2035, 1993.

[11] Deshpande, V. S., Ashby, M. F., \& Fleck, N. A., Acta Materialia, 49, 6, 1035- 1040, $2001 b$.

[12] Bhattacharya, K., Oxford series on materials modeling, vol. 2. New York: Oxford University Press, 2003.

[13] Grosso, G., \& Pastori-Parravincini, G., London: Academic Press, 2000.

[14] Jones, W., \& March, N. H., Vol. 1. London: Wiley-Interscience, 1973.

[15] Timoshenko, S. P., \& Young, D. H., McGraw-Hill, New York 1945.

[16] Langley, R. S., J. Sound Vib. 167, 377-381, 1993.

[17] Langley, R. S., Bardell, N. S., \& Ruivo, H. M., J. Sound Vib. 207, 521- 535, 1997.

[18] Brillouin, L., 2nd ed., Dover, New York, 1953.

[19] Born, M., \& Huang, K., Oxford: Clarendon Press, 1954.

[20] Maugin, G. A., Cambridge texts in applied mathematics. Cambridge: Cambridge University Press, 1992.

[21] Pitteri, M., \& Zanzotto, G., Boca Raton: Chapman \& Hall/CRC Press, 2003.

[22] Ericksen, J. L., edited by M. E. Gurtin. New York: Academic Press, 1984.

[23] Renton, J.D., 2nd ed., Horwood Publishing Limited, England, 2002.

[24] Horn, Roger A. and Johnson \& Charles R, Cambridge University Press, 1985.

[25] Horn, Roger A. \& Johnson, Charles R., Cambridge University Press, 1991.

[26] Strang G., 3rd ed., Wellesley-Cambridge Press, 1998.

[27] Hill, V. E., Boca Raton, Chapman \& Hall/CRC Press, 2000.

[28] Aschbacher, M., 2nd ed., Cambridge studies in advanced mathematics, 10, Cambridge, Cambridge University Press, 2000.

[29] James, G., \& Liebeck, M., 2nd ed., Cambridge: Cambridge University Press, 2001.

[30] Kuznetsov, E. N., Int. J. of Solids and Structures, 37, 15, pp. 2215-2223 April 2000.

[31] Kuznetsov, E.N., Int. J. of Solids and Structures 34, pp. 3657-3671, 1997. 